\title{
Germinal Center B-Lymphocyte
}

National Cancer Institute

\section{Source}

National Cancer Institute. Germinal Center B-Lymphocyte. NCI Thesaurus. Code C38335.

A mature B-lymphocyte that is present in the geminal center of a lymphoid follicle. 